\title{
EASE OF INSERTION, MAINTENANCE OF AIRWAY AND RISK OF INJURY ARE SIMILAR BETWEEN THE LMA-PROSEAL AND THE LMA-CLASSIC IN CHILDREN
}

\section{Ch Anil Kumar ${ }^{1}$, D Sandeep Kumar ${ }^{1}$, Srinivas ${ }^{2}$, Shanti Raj *3.}

${ }^{1}$ Assistant professor in Anaesthesia, Viswa Bharthi Medical College, Kurnool, Andhra Pradesh, India.

${ }^{2}$ Senior Resident in Anaesthesia, Viswa Bharthi Medical College, Kurnool, Andhra Pradesh, India.

${ }^{*}$ Associate professor in Anaesthesia, Viswa Bharthi Medical College, Kurnool, Andhra Pradesh, India.

\section{ABSTRACT}

Background: The LMA-Classic is a first generation supraglottic airway device, with largest evidence base for efficacy and safety, and is considered benchmark against which newer LMA are judged. The LMA-ProSeal is a second generation supraglottic airway device with modified cuff and drainage tube, designed for better seal with both the respiratory and gastrointestinal tracts, notwithstanding the access to the alimentary tract. This study compared is planned to compare efficacy of the LMA-Classic and LMA-ProSeal in children undergoing elective surgery under general anaesthesia.

Materials and method: This comparative study was done during the period of 01-Jan - 2018 to 30-aug-2018 in Viswa Bharthi Institute of Medical Sciences in the Department of Anaesthesiology with 40 ASA 1-2 children undergoing circumcision, herniotomy and orchiopexy were included. The patients were randomly assigned to size 2.5 LMA-ProSeal or 2.5 LMA-Classic groups for airway management. We assessed success rates at first attempt of insertion, airway sealing pressure, maintenance of airway and postoperative complications.

Results: There was no statistical difference between two groups for the success rates at first attempt of insertion and maintenance of airway but sealing pressure was significantly high in the LMA-ProSeal group. Regarding postoperative complication like injury to lip-teeth-tongue, blood staining and cough or laryngospasm were also not significant.

Conclusion: the ease of insertion, maintenance of airway and risk of injury are similar between the LMA-ProSeal and the LMA-Classic in children.

KEY WORDS: Paediatric anaesthesia, Equipment, Laryngeal mask airway.

Address for correspondence: Dr. Shanti Raj, Associate professor in Anaesthesia, Viswa Bharthi Medical College, Kurnool, Andhra Pradesh, India.

E-Mail: shantirajgunna123@gmail.com, thalithakumidhanya@gmail.com

\begin{tabular}{|c|c|c|}
\hline \multicolumn{3}{|c|}{ Online Access and Article Informtaion } \\
\hline \multirow{2}{*}{$\begin{array}{l}\text { Quick Response code } \\
\text { DOI: } 10.16965 / \text { ijims.2018.135 }\end{array}$} & \multicolumn{2}{|c|}{$\begin{array}{l}\text { International Journal of Integrative Medical Sciences } \\
\text { ISSN (P): } 2394 \text { - 6318. ISSN (E): } 2394-4137 \\
\text { www.imedsciences.com }\end{array}$} \\
\hline & $\begin{array}{l}\text { Received: 29-10-2018 } \\
\text { Reviewed: } 29-10-2018\end{array}$ & $\begin{array}{l}\text { Accepted: 24-11-2018 } \\
\text { Published: 25-12-2018 }\end{array}$ \\
\hline Source of Funding: Self & \multicolumn{2}{|c|}{ Conflicts of interest: None } \\
\hline
\end{tabular}

\section{BACKGROUND}

The Laryngeal Mask Airway (LMA) is a supraglottic airway device designed to maintain a clear airway, which sits outside of and creates a seal around the larynx. It is relatively non- invasive as compared to endotracheal intubation and in scenarios where endotracheal intubation is not mandatory; LMA has emerged as a formidable choice over endotracheal intubation [1]. Compared with the face mask, the 
LMA allows for a more "hands-free approach" to airway management [2]. In difficult airway management, LMA can bypass obstruction at supraglottic level and allow rescue oxygenation and ventilation, provided that mouth opening is sufficient [3].

The Laryngeal Mask Airway (LMA) is a supraglottic airway device designed to maintain a clear airway, which sits outside of and creates a seal around the larynx. It is relatively non- invasive as compared to endotracheal intubation and in scenarios where endotracheal intubation is not mandatory; LMA has emerged as a formidable choice over endotracheal intubation [1]. Compared with the face mask, the LMA allows for a more "hands-free approach" to airway management [2]. In difficult airway management, LMA can bypass obstruction at supraglottic level and allow rescue oxygenation and ventilation, provided that mouth opening is sufficient [3].

The LMA-Classic is a first generation supraglottic airway device, with largest evidence base for efficacy and safety, and is considered benchmark against which newer LMA are judged [1]. However, use of positive pressure ventilation and the associated gastric insufflations are a limitation of its use [4]. Brain invented LMA-Classic in 1983, and was introduced into pediatric anaesthesia practice in 1988. Since then, the LMAClassic has found widespread acceptance in Pediatric Anesthesia Practice [1,5]. However, the limitation of the LMA-Classic for use during the positive pressure ventilation and the associated gastric insufflations, led to the refinement of the design of the LMA-Classic leading to invention of the LMA-ProSeal by Brain et al in 2000 [6]. The LMA-ProSeal is a second generation supraglottic airway device with modified cuff and drainage tube, designed for better seal with both the respiratory and gastrointestinal tracts, notwithstanding the access to the alimentary tract [6-8]. The present study is planned to compare efficacy of the LMA-Classic and LMA-ProSeal in children undergoing elective surgery under general anaesthesia.

\section{MATERIALS AND METHODS}

The study will be done in the vishwabharathi Institute of Medical Sciences in the Department of Anaesthesiology and Critical Care for period from 01-Jan - 2018 to 30-aug-2018. After approval by institutional human studies committee and parental consent 40 ASA physical status 1-2 paediatric patients (aged 3-6 yr, weight $20-30 \mathrm{~kg}$ ) undergoing circumcision, herniotomy and orchiopexy were included in the study. Patient with lung disease, known airway problems, upper respiratory tract symptoms or any condition that increases the risk of gastrooesophageal regurgitation were excluded from the study. After enrolment, the patients were randomly assigned to a size 2.5 LMA-ProSeal group or a 2.5 LMA-Classic group for airway management using the sealed envelope method with 20 subjects in each group.

Anaesthesia Protocol: All patients were premeditated with oral diazepam $0.5 \mathrm{mg} / \mathrm{kg}$ or midazolam $0.3 \mathrm{mg} / \mathrm{kg} 1$ hour before induction of anaesthesia. After standard monitoring devices had been applied anaesthesia was induced by inhalation of nitrous oxide, oxygen and halothane. Once an adequate depth of anaesthesia had been achieved, each device was inserted by an experienced anaesthetist who had used the LMA-Classic more than 100 times and the LMA-ProSeal more than 20 times with the index finger insertion technique as per manufacturer's instructions. Both devices were fixed by taping the tube over chin and the cuff was inflated with air according to size.

An effective airway was judged by a squarewave capnograph trace, normal thoracoabdominal movement and inaudibility of stridor. If an effective airway could not be achieved, the device was removed and three attempts were permitted before failure of insertion was recorded. If the three attempts were unsuccessful, either an alternative device was inserted or the trachea was intubated. The numbers of insertion attempts were recorded. The sealing pressure was determined by closing the expiratory valve of the breathing system at a fixed gas flow of 3 Litre/min noting the airway pressure (maximum allowed was $40 \mathrm{~cm}$ of $\mathrm{H} 2 \mathrm{O}$ ) at which equilibrium was reached. At this time, gas leakage was determined at mouth (audible), the stomach (epigastric auscultation), or the drainage tube (bubbling of the lubricant placed on the proximal end of the drainage tube). In the 
LMA-ProSeal group only a lubricated 10-French gastric tube was inserted through the drainage tube. Adequate depth was maintained throughout the surgical period. At the end of the surgical period anaesthesia was discontinued and the device was removed. Postoperative blood staining of the LMA, injury to surrounding structure and cough or laryngospasm was recorded after removal of the device.
Table 1: Distribution of demography and clinical data $(\mathrm{N}=40)$.

\begin{tabular}{|c|c|c|}
\hline & $\begin{array}{c}\text { LMA-Classic } \\
(\mathbf{n = 2 0})\end{array}$ & $\begin{array}{c}\text { LMA-ProSeal } \\
(\mathbf{n = 2 0})\end{array}$ \\
\hline Age (3-6 yrs.) & 4 & 5 \\
\hline Weight $\mathbf{( k g )}$ & 24 & 25 \\
\hline Height $(\mathbf{c m})$ & 95 & 96 \\
\hline Circumcision & 15 & 15 \\
\hline Herniotomy & 3 & 3 \\
\hline Orchiopexy & 2 & 2 \\
\hline
\end{tabular}

Table 2: Comparison between LMA-Classic and LMA-ProSeal.

\begin{tabular}{|c|c|c|c|c|}
\hline & & $\begin{array}{c}\text { LMA-Classic } \\
(n=20)\end{array}$ & $\begin{array}{l}\text { LMA-ProSeal } \\
\quad(n=20)\end{array}$ & p-value \\
\hline \multirow{2}{*}{ Attempt at insertion } & 1st & 19 (95\%) & $18(90 \%)$ & \multirow{2}{*}{$0.999 * *$} \\
\hline & $2^{\text {nd }}$ or 3 rd & $1(5 \%)$ & $2(10 \%)$ & \\
\hline Seal pressure (cm H2O) & Mean \pm SD & $19.0 \pm 1.26$ & $20.0 \pm 1.26$ & $0.016 *$ \\
\hline \multirow{3}{*}{ Airway maintenance Clear } & Clear & $18(90 \%)$ & $19(95 \%)$ & $0.999 * *$ \\
\hline & Partial obstruction & $2(10 \%)$ & $1(5 \%)$ & \\
\hline & Complete obstruction & $q_{i}-$ & - & - \\
\hline \multirow{3}{*}{ Complication } & Cough or laryngospasm & $1(5 \%)$ & $2(10 \%)$ & $0.999 * *$ \\
\hline & Blood staining & $-\infty$ & - & - \\
\hline & Injury to lip \& tongue & 3 & - & - \\
\hline
\end{tabular}

*Unpaired $t$ test was done to measure the level of significance. ${ }^{* *}$ Fisher's exact test was done to measure the level of significance.

\section{DISCUSSION}

There was apparently no difference between the two groups with respect to demographic variables and regarding types of surgery same number of subjects were allocated in two groups for three different categories (Table I). In all patients an LMA was inserted within three attempts. The success rate at first attempt of insertion were 19/20 (95\%) for the LMA-Classic and $18 / 20$ (90\%) for the LMA-ProSeal. Regarding maintenance of airway partial obstruction is slightly more in the LMA-Classic than the LMAProSeal but which was not significant (Table II). Among complications cough or laryngospasm is slightly more in the LMA-ProSeal than the LMAClassic but which was not significant (Table II). Injuries to lip, tongue or blood staining were not detected in either group. But airway sealing pressure differed between two groups (Table II). In comparison between the two groups data were analyzed with the unpaired t-test. Unless otherwise stated data are presented as mean (SD). Significance was taken as $p<0.05$.

We found that ease of insertion and airway maintenance was similar in both LMA-ProSeal and the LMA-Classic in children. Several factors may have contributed to these findings. The main factor is probably the lack of rear cuff. In practice, when we deflate the cuff of the LMA-ProSeal completely, a fold occurs which may prevent smooth insertion of the device. The LMA-ProSeal of size 2.5 does not have a rear cuff; therefore no fold occurs. Another factor may be due to the airway tube and the drainage tube linings being side by side. This prevents rotation of the airway tube during insertion, especially in the narrow oral space in children, impeding digital positioning.

In similar studies showed that: In randomized crossover study by K. Goldmann et al, in which thirty anaesthetized, non-paralyzed children were randomly assessed with size 2 LMAProSeal and LMA-Classic, ease of insertion and quality of initial airway were similar for both devices. However, air entry into the stomach occurred more frequently with the LMA-Classic [9]. Shimbori H. et al randomized sixty children undergoing herniorrhaphy, orchiopexy or myringotomy to size 2 LMA-Classic or size 2 LMAProSeal groups for airway management. There was no statistical difference between the two 
groups for the success rates at first attempt of insertion. However the LMA-Classic had a higher rate of postoperative blood staining on the device, but there was no tongue-lip-dental trauma or hoarseness in either group [10].

Previous studies have reported success rates of LMA insertion in children of 67-99\%,8-12 which are comparable with our studies of $90-95 \%$. The difference in the rates may result from the different definitions of successful insertion and insertion technique. But an Indian study by Pravesh Kanthed et al, found the ease of insertion and the number of attempts at insertion were comparable between LMA-ProSeal and LMA-Classic. There was no difference in pharyngolaryngeal morbidity and no incidence of regurgitation or aspiration between LMA-ProSeal and LMA-Classic. Also, the LMAProSeal offered high reliability of gastric tube placement [8].

K. Goldmann et al, with size 21/2 LMA, in their randomized crossover study, had similar ease of insertion and first-attempt insertion success rate for LMA-ProSeal and LMA-Classic. However, The LMA-ProSeal had better quality of insertion. Air entry into the stomach was more common with the LMA-Classic [4]. In a randomized, non-crossover study by Lopez-Gil $\mathrm{M}$ et al, LMA-ProSeal and LMA-Classic had similar ease of insertion, fibreoptic position, and frequency of mucosal trauma. Also, there were no differences in performance among the sizes 2, 2.5, and 3 of either LMA-ProSeal or LMA-Classic [7]. These findings contrast with those described in adults. Brimacombe and colleagues presumed that the difficulties were caused by the larger cuff impeding digital intra-oral positioning and propulsion into the pharynx $[11,12]$. As it has been reported that the LMA-ProSeal provides a better airway seal than the LMA-Classic in adults, similar observation has been made in this study regarding the usage of two devices in children. Though the paediatric LMA-ProSeal lacks a rear cuff, we found that seal pressure was statistically higher in LMA-ProSeal group which might be due to our small sample size. Airway maintenance was similar in both the groups. Several reports suggested that better sealing pressure in LMA-ProSeal is mainly due the back cuff [11-13]. The lack of back cuff in
2.5 LMA-ProSeal means that it could not form a better seal than the LMA-Classic. In 1999, LopezGil and colleagues studied a prototype of the LMA-ProSeal for children, which had a rear cuff [14].

They stated that sealing pressure was over 40 $\mathrm{cm} \mathrm{H} 2 \mathrm{O}$ in all cases. This confirms the importance of rear cuff in airway seal pressure. In our study, the sealing pressure was measured by closing the expiratory valve of the circle system at a fixed fresh gas flow of $3 \mathrm{~L} / \mathrm{min}$ until airway pressure reached a steady value. Lopez-Gil and colleagues compared four kinds of measurements of the airway sealing pressure which involved detection of an audible noise by listening over the mouth, detection of an exhaled carbon dioxide by placing a gas sampling line for the capnograph inside the mouth, detection of a steady value airway pressure while occluding the expiratory valve of the circle system and detection of an audible noise using a stethoscope placed just lateral to the thyroid cartilage [15]. A limitation of our study is that the data were collected by an unblended observer.

\section{CONCLUSION}

There was no statistical difference between two groups for the success rates at first attempt of insertion and maintenance of airway but sealing pressure was significantly high in the LMA-ProSeal group. Regarding postoperative complication like injury to lip-teeth-tongue, blood staining and cough or laryngospasm were also not significant. There is no difference between the LMA-Classic and the LMA-ProSeal concerning case of insertion and airway maintenance in children.

\section{REFERENCES}

[1]. Michelle C. White, Tim M. Cook, Peter A. Stoddart. A critique of elective pediatric supraglottic airway devices. Pediatric Anesthesia 200919 (Suppl. 1): 55-65.

[2]. Efrat R, Kadari A, Katz. The laryngeal mask airway in pediatric anesthesia: experience with 120 patients undergoing elective groin surgery. J Pediatr Surg. 1994 Feb;29(2):206-8.

[3]. Bhavesh Patel, Robert Bingham. Laryngeal mask airway and other supraglottic airway devices in paediatric practice Contin Educ Anaesth Crit Care Pain February 1, 2009 9:6-9. 
[4]. Goldmann K, Jakob C. A randomized crossover comparison of the size $21 / 2$ laryngeal mask airway ProSeal versus laryngeal mask airway-Classic in pediatric patients. Anesth Analg. 2005 Jun;100(6):1605-10.

[5]. Brain Al. The development of the Laryngeal Mask-a brief history of the invention, early clinical studies and experimental work from which the Laryngeal Mask evolved. Eur J Anaesthesiol Suppl 1991; 4: 517.

[6]. Brain Al, Verghese C, Strube PJ. The LMA ProSeal - a laryngeal mask with an oesophageal vent. $\mathrm{Br} J$ Anaesth. 2000 ; 84 (5): 650-4.

[7]. Lopez-Gil M, Brimacombe J, Garcia G. A randomized non-crossover study comparing the ProSeal and Classic laryngeal mask airway in anaesthetized children. Br J Anaesth. 2005 Dec;95(6):827-30.

[8]. Kanthed P, Sharma B, Sood J, Kumra VP. Comparison of LMA-ProSeal ${ }^{\text {TM }}$ with LMA Classic ${ }^{\text {TM }}$ in Anaesthetised Paralysed Children. Indian J Anaesth 2008;52:44.

[9]. K. Goldmann, C. Jakob. Size 2 ProSeal $^{\mathrm{TM}}$ laryngeal mask airway: a randomized, crossover investigation with the standard laryngeal mask airway in paediatric patients. Br J Anaesth. 2017;94(3):385389.

[10].H. Shimbori, K. Ono, T. Miwa, N. Morimura, M. Noguchi, K. Hiroki. Comparison of the LMA-ProSeal ${ }^{\mathrm{TM}}$ and LMA-Classic ${ }^{\mathrm{TM}}$ in children. Br J Anaesth. 93(4): 528-531.
[11]. Brimacombe J, Keller C. The ProSeal Laryngeal Mask Airway: A Randomized, Crossover Study with the Standard Laryngeal Mask Airway in Paralyzed, Anaesthetized Patients. Anesthesiology. 2000;93:104109.

[12].Brimacombe J, Keller C, Fullekrug B, Agrò F, Rosenblatt W, Dierdorf SF, et al. A Multicenter Study Comparing the ProSeal and Classic Laryngeal Mask Airway in Anesthetized, Nonparalyzed Patients. Anesthesiology. 2002;96:289-95.

[13]. Cook TM, Nolan JP, Verghese C, Strube PJ, Lees M, Millar JM, et al. Randomized Crossover Comparison of the Proseal with the Classic Laryngeal Mask Airway in Unparalyzed Anaesthetized Patients. Br J Anaesth. 2002;88:527-33.

[14]. Lopez-Gil M, Brimacombe J. Pulmonary Evaluation of a New Prototype Laryngeal Mask Airway in Children. Br J Anaesthe. 1999;82:132-34.

[15]. Lopez-Gil M, Brimacombe J, Keller C. A Comparison of Four Methods for Assessing Oropharyngeal Leak Pressure with the Laryngeal Mask Airway in Pediatric Patients. Pediatric Anaesth. 2001;11:319-21.

How to cite this article: Ch Anil Kumar, D Sandeep Kumar, Srinivas, Shanti Raj. EASE OF INSERTION, MAINTENANCE OF AIRWAY AND RISK OF INJURY ARE SIMILAR BETWEEN THE LMA-PROSEAL AND THE LMACLASSIC IN CHILDREN. Int J Intg Med Sci 2018;5(9):741-745. DOI: 10.16965/ijims.2018.135 